\title{
Uterine Inversion: Management Protocol with Literature Review
}

Pratiksha Gupta, Alka Sehgal, Sonil Prasbhakar, Anju Huria, Ram-ji. Lal Sahu

Department of Gynae and Obstetrics

Government Medical College, Chandigarh, India

'M. M. Medical College, Ambala

\begin{abstract}
Uterine inversion is an introflexion of parietes uteri, which takes place usually during the third stage of labor or sometimes during the puerperium. Inversion has been categorized in three degrees depending on the amount of introversion. Acute uterine inversion is a rare but life threatening complication of the third stage of labour because of post partum haemorrhage shock or both. The incidence in literature varies widely, quoted between 1 in 2000 to 1 in 50000 deliveries. Prompt and aggressive management can lower maternal morbidity and mortality. Etiology of uterine inversion is unclear. Despite the many conditions associated with uterine inversion risk assessment is often lacking making the condition usually unexpected at the time of presentation. Optimal treatment for inversion includes aggressive management, prompt diagnosis and shock management. This article defines diagnostic tools, various management protocols.
\end{abstract}

Key words: Acute uterine inversion, complication of third stage of labour, post-partum haemorrhage, shock

\section{Introduction}

Severe post-partum haemorrhage and shock result from the uterine fundus collapsing. If unrecognized, this obstetric emergency could cause serious morbidity or death.

Uterine inversion is reported to occur at a rate of 1 case per 2000-23,000 deliveries ${ }^{1}$. This wide range reflects differences in recording methods in patient populations and perhaps in routine obstetric techniques. As examples of recent literature, in the 24-year series reported by Baskett involving 125,081 deliveries, the incidence was 1 in 3737 for vaginal deliveries and 1 in 1860 for caesarean deliveries. ${ }^{2}$ In the series described by Hussain et $\mathrm{al}^{3}$ and Shah-Hosseini ${ }^{4}$ et al, puerperal inversions occurred in 1 in 1584 and 1 in 4345 deliveries, respectively, while Abouleish ${ }^{5}$ reported an incidence of 1 in 3643. This condition, while uncommon, is far from rare. Most commonly, the frequency is reported to be approximately 1 in 2000 deliveries; however, a range of 1 in 4000 to 1 in several hundred thousand can be found. ${ }^{6,7}$
Maternal mortality has been reported to be as high as $15 \%{ }^{8}$

\section{Classification}

Uterine inversion may occur in the immediate postpartum period or, less frequently, during the puerperium. Inversion may be described as recent/acute ( $<30 \mathrm{~d}$ after delivery) or chronic ( $>30 \mathrm{~d}$ after delivery).Recent inversion may further be classified as - Acute inversions which occur within the first 24 hours after delivery, whereas inversions occurring more than 24 hours after delivery but before the 30 th postpartum day are termed subacute. ${ }^{1}$ Uterine inversion is classified by the degree of inversion and by the time of onset . The uterine fundus that has inverted and lies within the endometrial cavity without extending beyond the external os is called an incomplete inversion. Complete inversion describes an inverted fundus that extends beyond the external os. ${ }^{9} \mathrm{~A}$ prolapsed inversion is one in which the inverted uterine fundus extends beyond the vaginal introitus. ${ }^{8}$ A total inversion, which is usually

Corresspondence

Dr Pratiksha Gupta, M. D.

Asst. Professor

Department of Gynae and obstetrics

Government Medical College, Chandigarh.

Tel. no- 09646121584

Email-drpratiksha@gmail.com 
nonpuerperal and tumor related, is associated with inversion of the uterus and vaginal wall . ${ }^{9}$ Classified in terms of onset of the inversion, acute describes the event occurring before contraction of the cervical ring. If the cervical ring has contracted, a subacute inversion has occurred. The inversion is classified as chronic if 4 weeks have elapsed before the event., ${ }^{4,9,10}$

\section{Etiology}

Etiology of uterine inversion is unclear. The most likely cause is strong traction on the umbilical cord, particularly when the placenta has a fundal implantation, during the third stage of labor. ${ }^{11}$ Other factors might include excessive fundal pressure; relaxed uterus, lower uterine segment and cervix; placenta accreta, particularly involving the uterine fundus; short umbilical cord; congenital weakness or anomalies of the uterus; and antepartum use of magnesium sulfate or oxytocin..$^{4,11,12,13}$ Some authors also suggest primiparity and rapid emptying of the uterus after prolonged distention as possible predisposing factors. 4,9,10. Uterine inversion and placenta accreta can both be fatal complications either alone or in combination ${ }^{14}$. Despite the many conditions associated with uterine inversion risk assessment is often lacking making the condition usually unexpected at the time of presentation. The association between abnormal placentation such as placenta accreta and uterine inversion is well supported ${ }^{13}$. Hence antenatal evaluation and risk assessment for placenta accreta might be useful ${ }^{15}$. Prenatal ultrasound reported sensitivity of $94 \%$ and specificity of $79 \%$ for placenta accreta but offers no more than provisional diagnostic probability statement ${ }^{16}$. If clinically or sonographically the patient is antenatally suspected to be at risk of placenta accreta appropriate management options should be considered in advance ${ }^{16}$.

\section{Diagnosis}

Diagnosis is usually established clinically except in rare cases. Patients will usually present with early onset post partum haemoirrhage with an appearance of a vaginal mass subsequently followed by maternal cardiovascular collapse. The initial event usually encountered is the protrusion of a large, dark red, polypoid mass through the vagina either accompanying or following the delivery of the placenta. In approximately $60-70 \%$ of cases the placenta, remains attached initially at the time of inversion ${ }^{1}$. Some degree of acute maternal cardiovascular decompensation occurs in all cases of uterine inversion with the extent of shock being disproportionate to the observed blood loss. This can be explained by the stretching of the broad ligament or compression of the ovaries as they are drawn together results in a parasympathetic reflex, which contributes to the acute symptoms (neurogenic shock) ${ }^{17}$. The prompt uterine replacement combined with vigorous fluid resuscitation including blood transfusion as required, reverses the hypotension. The neurogenic component of shock postulated to be due to parasympathetic effect of traction on ligaments and may be associated with bradycardia. This description is not well supported by literature may mislead emergency management. ${ }^{17,18}$ Profuse bleeding, absence of uterine fundus, or an obvious defect of the fundus on abdominal examination, as well as evidence of shock with severe hypotension, provide the clinician with diagnostic clues ${ }^{4,19}$. Although clinical symptoms will provide the diagnosis in most cases, radiographic methods to diagnose inversion have also been described in the literature in cases wherein the inversion is retained entirely within the uterus and has not passed through the cervix into the birth canal. Hsieh and Lee ${ }^{20}$ describe the sonographic findings of uterine inversion discovered incidentally in an acute incident. In the transverse images was visualized "a hyperechoic mass in the vagina with a central hypoechoic H-shaped cavity." Longitudinal images showed a U-shaped "depressed longitudinal groove from the uterine fundus to the center of the inverted part." Magnetic resonance imaging (MRI) of inversion has also been reported. The appearance of the uterus is similar to that found in sonographic imaging; however, MRI findings are much more conspicuous. ${ }^{8}$ Thus radiographic imaging can help when the diagnosis is uncertain after examination, and the patient is sufficiently stable clinically to undergo such evaluation.

\section{Chronic uterine inversion}

Chronic uterine inversion may be discovered as late as 14 weeks postpartum ${ }^{1}$ presenting with a variety of vague symptoms, including persistent vaginal bleeding or discharge, as well as symptoms of low back pain or pelvic pressure. Such symptoms may also be accompanied by malaise or low-grade temperature elevation. At least once case was complicated by a mesenteric vein thrombosis ${ }^{21}$. Differential diagnosis of uterine inversion include-Prolapse of a uterine tumor or a large cervical polyp, passage of previously unsuspected secundines or a succenturiate lobe, Gestational trophoblastic disease,foreign body in the vagina,occult laceration of the genital tract,severe uterine atony,unanticipated delivery of a second twin.

\section{Treatment}

Optimal treatment for inversion includes aggressive administration of parenteral fluids, transfusion of blood and blood products, and prompt uterine replacement followed by the administration of potent uterotonics. The approach to treatment should follow a logical progression that is to manage shock aggressively to be followed by an attempt at prompt replacement of the uterus. Replacement is further aided by administering tocolysis to promote uterine relaxation 
followed by uterotonics. The longer the delay in treatment, the greater is the risk of blood loss and maternal cardiovascular collapse and its serious sequelae. Also, as the delay lengthens, the lower uterine segment and/or cervix increasingly contracts, rendering the prolapsed fundus more edematous than before. Thus, delay simply makes replacement progressively more difficult.Uterine replacement can be surprisingly easy when performed immediately and the need to resort to various complex manipulations to return the uterus to its anatomic location can be avoided. Chances of immediate reduction is between 22-43\% ${ }^{5,22,23} \mathrm{~A}$ potential exception to this rule is the case of inversion is when the placenta remains attached to the uterus. If immediate reduction is unsuccessful further attempts should wait till patient is hemodynamically stable $^{24}$.Once inversion is recognized, all oxytocic agents should be withheld until correction has been established. Manual correction of inversion through the vagina, known as the Johnson maneuver, consists of pushing the inverted fundus through the cervical ring with pressure directed toward the umbilicus. ${ }^{12}$ Controversy exists as mentioned earlier about whether the placenta should be removed before repositioning the uterus. It is commonly suggested that removal of the placenta before correction will result in increased blood loss and worsening hemodynamics. ${ }^{7,12}$ To assist the clinician in maneuvering, myometrial relaxation is implemented by various medications. Most commonly used is magnesium sulfate or terbutaline, because they are readily available on most obstetric floors. These medications relax the uterus as well as the cervical contraction ring. Tocolysis can be achieved by general anaesthesia ${ }^{25}$, beta 2 adrenergic agonists ${ }^{23,26,27}$, nitroglycerine ${ }^{28}$ or magnesium sulphate ${ }^{29}$. General anaesthesia with a potent inhalational anaesthetic has been recommended to relax the uterus ${ }^{25}$. However general anaesthesia exposes the mother to the possibility of aspiration and hypoxic brain injury or maternal death if there is inability to intubate trachea ${ }^{5}$. The use of high concentration of a potent inhalational anaesthetic to relax the uterus depresses the cardiovascular system at the time when the patient is hemodynamically unstable.It takes longer to achieve a high blood concentration of inhalational anaesthetic than i.v injection of terbutaline which causes relaxation in less than 2 minutes. In a series reported by Abouleish et al ${ }^{5}$ in order to find an alternative to general anaesthesia terbutaline was studied and found to be successful in $63 \%$ of the patients ${ }^{5}$. Magnesium sulphate also considered as a tocolytic agent but takes at least 10 minutes to be effective ${ }^{29}$. IV dose of $4 \mathrm{G}$ magnesium sulphate is recommended ${ }^{6,30}$. Nitroglycerin has been used for extraction of retained placenta, external version and uterine inversion ${ }^{28}$. Benefits cited for the use of low-dose nitroglycerin include quicker onset of uterine relaxation; quick dissipation of the effect, obviating the need for reversal; and less effect on hemodynamics than magnesium sulfate ${ }^{31}$. In the event that correction is not established with tocolytic agents, general anesthesia with halothane may be induced to provide uterine relaxation. This approach can be particularly useful when the patient is hemodynamically unstable, because halothane anesthesia has fewer potential adverse effects on hemodynamics than do the $\beta$-adrenergic tocolytics. ${ }^{19}$ Johnson originally described in 1949 what is currently the treatment of choice ${ }^{1}$. In this procedure, after the administration of the tocolytic and an analgesic, the operator's hand is placed in the vagina, with the palm cupping the inverted fundus. The uterus is then firmly and promptly lifted upward in the pelvic curve through the pelvis and into the abdominal cavity to the level of the umbilicus. This manipulation forces the uterine ligaments to stretch. When the inverted mass is pushed upward, the uterus typically reverts promptly, and the fundus returns to its anatomic position. If reversion is successful, the uterus is held in place for several minutes. Parenteral uterotonics are then administered to firm the myometrium. To facilitate uterine replacement tocolytics/ general anaesthetics are used as discussed earlier. Hydrostatic pressure, is another method used to reposite the uterus when inversion has occurred. In this method, first described by O'Sullivan in the British Medical Journal in 1945, a bag of warmed fluid is hung on a pole used for intravenous fluids above the level of the patient and allowed to flow, via tubing, into the vagina. The pressure of the water, held in place by the clinician's hands, results in correction of the inversion. Momani and Hassan ${ }^{7}$ reported successful correction in five cases of inversion within a 7-year period using this method. More recently, Ogueh and Ayida ${ }^{32}$ described a new technique of hydrostatic pressure. Citing difficulty in maintaining an adequate water seal to generate the pressure required, the authors suggest attaching the intravenous tubing to a silicone cup used in vacuum extraction. By placing the cup within the vagina, an excellent seal is created, and adequate hydrostatic pressure for inversion correction is thus produced. Although success with this technique is cited in the literature, there has been no discussion of the theoretical risk of air or amniotic fluid embolus. If 2 or more attempts at manual replacement are unsuccessful despite adequate tocolysis and analgesia, a surgical procedure is indicated. An abdominal approach for uterine replacement is favored. The favored transabdominal technique is a modification of the procedure Huntington originally described in 1921. The Huntington procedure requires a laparotomy to locate the cup of the uterus formed by the inversion. Clamps are placed in the cup of the inversion below the cervical ring, and gentle upward traction is applied. Repeated clamping and traction continues until the inversion is corrected. In the 
unusual instance that the Huntington operation is unsuccessful, the more extensive Haultain procedure is required. In the Haultain procedure, an incision is made in the posterior portion of the inversion ring, again through the abdomen, to increase the size of the ring and allow repositioning of the uterus. This is usually necessary only when inversion is chronic or when tocolysis fails to adequately relax the lower segment. Regardless of the procedure used, immediate uterine atony is common after repositioning, and prompt reinversion may occur. Close observation for this complication is mandatory. Also recommended is the administration of 15-methyl F2alpha prostaglandin, high-dose oxytocin, parenteral methylergonovine maleate, or misoprostol per rectum. If magnesium sulfate was administered as a tocolytic, calcium can be administered parenterally to reverse the tocolytic effect. Recurrent risk and counselling. In the 172 case series reported by van Vugt ${ }^{33}$ references were made to a number of previous reports in which recurrences were reported. In 2002 report by Baskett et al of 40 cases of post partum inversion drawn from 125,081 births over 24 years, no recurrence was recorded in 26 subsequent deliveries. ${ }^{2}$ Time is an important factor in maternal mortality. In van Vugt's series derived from cases over many years, mortality was lowest when the condition was recognized promptly (within $30 \mathrm{~min}$ of delivery) and treatment was begun within 2 hours postpartum. ${ }^{33}$ With delay, mortalities were much more frequent. These facts emphasize the importance of celerity on the part of the birth attendants in establishing the diagnosis, replacing the uterus, and aggressively supporting the mother's condition. If a surgical procedure with hysterotomy incision(s) were required for uterine replacement, the woman should be counselled that a risk of uterine rupture in a subsequent gestation during labor is possible. In theory, if the incision in myometrium remains in the lower uterine segment, the rupture risk should approximate that for the usual anterior vertical hysterotomy incision occasionally used for caesarean delivery. The actual rupture risk is, unfortunately, unknown due to the paucity of data.

\section{Conclusion}

Prompt diagnosis of acute puerperal inversion immediately followed by manual reposition of the uterus or the application of hydrostatic method whenever possible can be life saving. However expertise surgical correction is further needed in cases of more resistant uterine inverversion that was either over looked or badly manipulated at the outset because of the misdiagnoses of the condition. Therefore the message would be to enable health workers / Traditional Birth Attendants to practice, active management of third stage of labour in order to help reduce the mishaps that is connected with mismanaged third stage of labour thereby reducing associated maternal mortality morbidity despite of aggressive managements that may be futile.

\section{References}

1. O' Grady John P et al. Malposition of the uterus. E Medicine, 2008

2. Baskett TF. Acute uterine inversion: a review of 40 cases. J Obstet Gynaecol Can. Dec 2002; 24(12):953-6. [Medline].

3. Hussain M, Jabeen T, Liaquat N, Noorani K, Bhutta SZ. Acute puerperal uterine inversion. J Coll Physicians Surg Pak. Apr 2004;14(4):2157. [Medline].

4. Shah-Hosseini R, Evrard JR. Puerperal uterine inversion. Obstet Gynecol. Apr 1989;73(4):56770. [Medline].

5. Abouleish E, Ali V, Joumaa B, Lopez M, Gupta D. Anaesthetic management of acute puerperal uterine inversion. Br J Anaesth. Oct 1995; 75(4):486-7. [Medline].

6. Catanzarite VA, Moffitt KD, Baker ML, Awadalla SG, Argubright KF, Perkins RP. New approaches to the management of acute puerperal uterine inversion. Obstet Gynecol 1986;68(3 Suppl):7S$10 \mathrm{~S}$.

7. Momani AW, Hassan A. Treatment of puerperal uterine inversion by the hydrostatic method: reports of five cases. Eur J Obstet Gynecol Reprod Biol 1989;32:281-5.

8. Lewin JS, Bryan PJ. MR imaging of uterine inversion. J Comput Assist Tomogr 1989;13:3579.

9. Salomon CG, Patel SK. Computed tomography of chronic nonpuerperal uterine inversion J Comput Assist Tomogr 1990;14:1024-6.

10. Zahn CM, Yeomans ER. Postpartum hemorrhage: placenta accreta, uterine inversion, and puerperal hematomas. Clin Obstet Gynecol 1990;33:422-31.

11. Cunningham FG, MacDonald PC, Gant NF, et al. Williams obstetrics. 20th ed. Stamford, Conn: Appleton \& Lange, 1997:767-9.

12. Kochenour NK. Intrapartum obstetric emergencies. Crit Care Clin 1991;7(4):851-64.

13. Hostetler DR, Bosworth MF: Uterine inversiona life- threatening emergency. J Am Board Fam Pract 2000, 13(2): 120-3.

14. Agarwal S, Minocha B, Dewan R: Uterine inversion and concomitant perforation following manual removal of placenta. Int J Gynaecolobstet 2005, 88(2): 144-145.

15. Finberg HJW: Placenta accreta prospective sonographic diagnosis in patients with placenta previa and prior cesarean section. J Ultrasound Med 1992, 11(7): 333-344. 
16. Armstrong CA, Harding S, Dickinson JE: Clinical aspects and conservative management of placenta accrete. Obstet and Gynaecol 2004, 6: 132-137.

17. Schneider MC, Hampl KF. Peripartum anaesthesia. In: Russell IF, Lyons G, eds. Clinical problems in Obstetric Anaesthesia. London: Chapman and Hall Medical, 1997; 50-51.

18. Beringer RM, Patteril M: Puerperal uterine inversion and shock. British J of Anaesthesia 2004,92(3):439-441.

19. Varner M. Postpartum hemorrhage. Crit Care Clin 1991;7:883-97.

20. Hsieh TT, Lee JD. Sonographic findings in acute puerperal uterine inversion. J Clin Ultrasound 1991; 19:306-9.

21. Livingston SL, Booker C, Kramer P, Dodson WC. Chronic uterine inversion at 14 weeks postpartum. Obstet Gynecol. Feb 2007;109(2 Pt2):555-7. [Medline]

22. Platt LD, Druzin ML. Acute puerperal inversion of the uterus. Am J Obstet Gynaecol 1981; 141:187190.

23. Brar HS, Greenspoon JS, Platt LD, Paul RH. Acute puerperal inversion. New approaches to management. Journal of reproductive Medicine 1989; 34: 173-177

24. Still DK. Post partum haemorrhage and other problems of the third stage. In: James DK, Steer PJ, Weiner CP, Gonik B, eds. High risk pregnancy and management options. London: WB Saunders Company Ltd, 1994; 1175-1177.

25. Datta S. Anaesthetic and obstetric management of high risk pregnancy. St Louis: Mosby Year Book, 1991; 129-130, 465-466

26. Kowacs BW, DeVore GR. Management of acute and subacute puerperal uterine inversion with terbutaline sulphate. American journal of obstetrics and gynaecology 1984; 150: 784-786

27. Clark SL. Use of ritodrine in uterine inversion. American journal of obstetrics and gynaecology 1985; 151: 705

28. Bahyi DA, Sherwood CD, Campbell CE. Intravenous nitroglycerin for uterine inversion. Journal of clinical anaesthesia 1992; 4: 487-488

29. Grossman RA.Magnesium sulphate for uterine inversion. Journal of reproductive medicine 1981; 26:261-262

30. Wendel PJ, Cox SM. Emergency obstetric management of uterine inversion. Obstet Gynaecol Clin North Am 1995; 22: 261-274.

31. Dayan SS, Schwalbe SS. The use of small-dose intravenous nitroglycerin in a case of uterine inversion. Anesth Analg 1996;82:1091-3.

32. Ogueh $\mathrm{O}$, Ayida G. Acute uterine inversion: a new technique of hydrostatic replacement. Br J Obstet Gynaecol 1997;104:951-2.

33. van Vugt PJ, Baudoin P, Blom VM, van Deursen CT. Inversio uteri puerperalis. Acta Obstet Gynecol Scand. 1981;60(4):353-62. [Medline] 